\title{
Postoperative osteopathic manipulative management of median sternotomy patients
}

\author{
JERRY L. DICKEY, DO
}

More than 250,000 patients yearly undergo coronary bypass graft surgery accomplished via the median sternotomy incision, an approach that has been gaining widespread acceptance. This surgical approach has been associated with a growing number of patients with structural complaints. This article describes a postoperative treatment protocol for improving healing and reducing musculoskeletal disability associated with such cardiac procedures and presents a logical sequence of treatment graded to the patient's changing condition and stage of healing. The author challenges the osteopathic medical profession to meet the opportunity that exists to educate patients and physicians about the benefits of osteopathic healthcare.

There has been increasing acceptance of the use of the median sternotomy approach in intrathoracic surgical procedures. It is the preferred approach for cardiac surgical procedures, and a growing number of thoracic sur-

\footnotetext{
Dr Dickey is chairman of the department of manipulative medicine, Texas College of Osteopathic Medicine, Fort Worth. He wrote this thesis as part of the requirement for Fellowship certification in the American Academy of Osteopathy.

Reprint requests to Jerry L. Dickey, DO, chairman, department of manipulative medicine, Texas College of Osteopathic Medicine, 3500 Camp Bowie Blvd, Fort Worth, TX 76107-2690.
}

geons are also using this incision in pulmonary procedures. ${ }^{1}$ Attendant to the more widespread use of this operative approach has been an increase in the number of patients who are being seen with structural complaints postoperatively. Current medical literature documents the more dramatic consequences but fails to relate more subtle changes in chest cage mechanics resulting from the use of this approach.

The purpose of this article is to suggest an osteopathic treatment regimen that addresses these alterations of anatomy and physiology.

\section{Incidence}

Although it is difficult to pinpoint the precise number of surgical procedures in which the median sternotomy approach is used, the number in the United States probably exceeds 250,000 per year. The sharp increase in coronary bypass graft procedures for the treatment of coronary insufficiency contributes the largest single group of patients undergoing median sternotomy. From 1979 to 1982, there was a $48 \%$ increase in the number of coronary bypass graft procedures ${ }^{2}$; in 1983 , there were a reported 191,000 such procedures performed in the United States. ${ }^{3}$ The increasing use of coronary bypass graft surgery, in addition to other open heart and pulmonary procedures, could push the number of median sternotomies to more than 400,000 per year in the near future. Such numbers behoove the osteopathic medical profession to develop treatment approaches that will address the postoperative structural needs of this enlarging population. 


\section{Common operative approach}

In the common operative technique for median sternotomy, the surgeon divides the skin with a rectilinear median incision from the suprasternal notch to below the xiphoid process and uses diathermy to separate the subcutaneous layers and achieve hemostasis. The surgeon then divides the sternum by use of an electric bone saw and covers the exposed edges with bone wax. ${ }^{4}$ Next, the operator retracts the sternum by use of an Ankeney retractor, with the upper end placed at the level of the second rib. For cardiac procedures, the pericardial sac is opened to expose the heart.

Following the surgical procedure, some surgeons close the pericardium, while others incompletely close the pericardium to allow better drainage. The cut sternal margins are approximated with four to six stainless steel figure-of-eight sutures. The superficial tissues usually are closed with cutaneous absorbable sutures. Pericardial, substernal, and pleural drainage tubes exit the chest below the xiphoid process.

\section{Common sequelae}

The common postoperative sequelae reported in the literature include wound dehiscence, substernal and pericardial infection, nonunion of the sternum, pericardial constriction, phrenic nerve injuries, rib fractures, and brachial plexus injuries. The last two sequelae are of particular interest because they are probably related.

Studies have placed the incidence of brachial plexus injuries as high as $23.5 \% .^{5}$ Vander Salm and associates ${ }^{5}$ performed median sternotomies on ten cadavers using the common surgical approach previously described. Seven of the ten cadavers sustained first rib fractures, with the fractured ends of the ribs often impaling the lower trunks of the brachial plexus. These investigators operated on an additional ten cadavers in which they placed the upper end of the Ankeney retractor at the level of the fourth rib instead of at the level of the second rib, and they observed no rib fractures. ${ }^{5}$ Such fractures usually are occult and are detected only by radioactive isotope bone scanning; many go unrecognized.

\section{Treatment plan}

All of the complications reported in the literature are readily observed in the immediate postoperative period. The literature fails to mention the structural and functional changes in chest cage mechanics that do not become evident until weeks, or even months, after surgery. Of interest to the osteopathic physician are hypomobility and somatic dysfunction of the thoracic vertebrae and rib cage, disruption of fascial patterns through the mediastinum, back pain (thoracic), and limited or distorted dysfunction of the diaphragm.

The treatment plan proposed here addresses these potential sequelae as they arise, starting with the preoperative phase and continuing through the immediate postoperative period to approximately 6 months postoperatively.

\section{Preoperative treatment plan}

Ideally, the osteopathic physician should assess the patient's rib cage function preoperatively, noting and recording symmetry or asymmetry and breathing patterns, including depth of respiration as a function of diaphragmatic excursion. Somatic dysfunction of the cervical vertebrae should be mobilized in preparation for intubation. Somatic dysfunction of the thoracic vertebrae and ribs should be treated to optimize tidal volume, and lumbar restriction should be improved to aid the excursion of the diaphragm. Unfortunately, all too often the osteopathic physician does not see the patient until after surgery, and so has not had the opportunity to assess preoperative function.

One should attempt to impress on the surgeon the importance of anatomically accurate approximation of the sawed sternal halves. The function of the sternal angle of Louis is vital to the proper function of the upper rib cage. It is not uncommon for surgeons to accept discrepancies of 0.25 inch when suturing the sternal halves. Such malalignment may well adversely affect sternal and rib cage mechanics.

\section{Early postoperative treatment plan}

Usually, it is not until the postoperative period that the osteopathic physician sees the pa- 


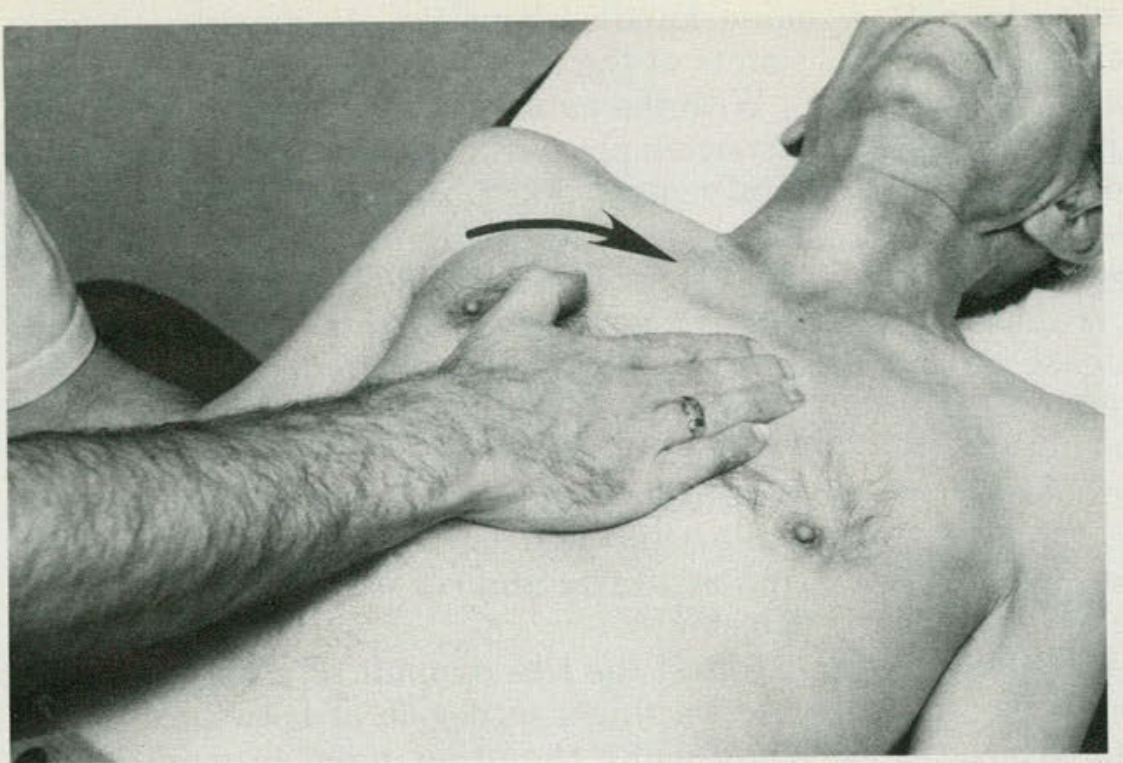

Figure 1. Testing fascial patterns of the chest. tient who has undergone median sternotomy. Ideally, one should begin assessment and treatment as soon as possible after the patient's dismissal from surgery and before there has been time for the sequelae associated with healing to develop. Certainly, a month postsurgery is not too soon to begin treatment.

The proposed treatment plan is based on the assumption that functional healing of the sternum is incomplete. Modifications in type of treatment and duration are made as healing continues. Since the majority of median sternotomy patients will have undergone coronary bypass graft surgery, it is likely that this patient population is in the sixth and seventh decades of life. Such patients likely will have chronic musculoskeletal problems and osteoarthritis that predate the surgical insult. Chronic obstructive pulmonary disease and other chronic disease states may further complicate the assessment.

The initial step is to repeat the evaluation previously described here for the preoperative assessment. Even if presurgical data are not available, postsurgical evaluation of breathing patterns and chest cage symmetry is helpful. The physician should lightly palpate the sternum to assess functional healing and the costochondral junctions to determine the degree of trauma sustained.
The upper four ribs usually are more severely injured as a result of the placement of the Ankeney retractor. Upper ribs normally have less mobility than lower ribs owing to their firmer attachment, posteriorly to the vertebrae and anteriorly to the sternum. The wide retraction necessary for the median sternotomy approach places more mechanical force on the upper than on the lower ribs. Intercostal muscle contraction is usually present, as is dysfunction and focal contraction of the diaphragm.

With the patient in the supine position, the physician evaluates the patterns of injury to the chest and sternum by placing one hand between the scapulas (Fig 1) while placing and resting the other hand on the skin in the midline of the sternum. Turning each hand independently in a clockwise, then counterclockwise direction, will reveal the tissue preference pattern. These findings should be recorded because they usually change through the course of treatment.

Treatment consists of gently encouraging both anterior and posterior tissue preference patterns and holding them until tissue tension relaxes. The physician should monitor changing tension states during the treatment phase and make modifications to maximize tissue relaxation. This fascial approach should be con- 
tinued on a weekly basis until the fascial patterns cease to change or are resolved.

The ideal pattern for the fascia of the chest would be to have equal excursion in both clockwise and counterclockwise rotations of the tissues; however, according to Zink and associates, ${ }^{6}$ most individuals have a preference pattern in these tissues. Treatment of these fascial patterns will commonly release recent strains and reveal older tissue preference patterns. Treatment may release strains and return the tissue to the ideal state. Most commonly, changing tissue patterns will assume an older strain pattern and will become static.

Next, the physician should cautiously approach the rib cage. One should refrain from high-velocity techniques because occult rib fractures are all too common in patients who have had median sternotomy. Function of the rib cage should be assessed both statically and dynamically. Because upper ribs predominate in

Figure 2. Evaluating chest cage compliance.

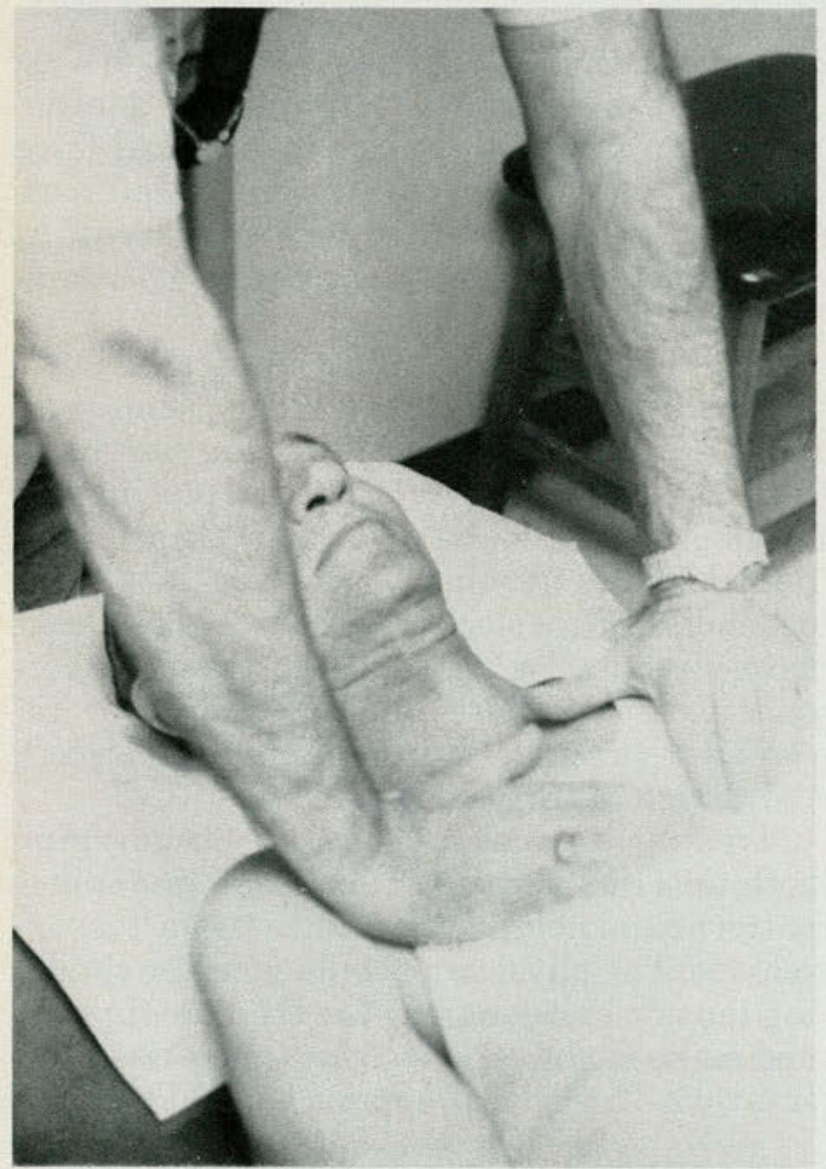

pump handle rib motion, the maximum excursion is at the anterior end.

With the patient in the supine position, the physician places flattened palms over the pectoral regions (Fig 2), directing alternating but equal pressure posteriorly through the anterior aspect of the chest wall into the second through sixth ribs. Asymmetry of compressibility indicates the need for more specific motion testing of each rib. A similar test is used for mid and lower ribs: The physician places flattened palms against the rib cage at the midaxillary line and applies alternating compression medially and records any asymmetry detected.

To test the ribs dynamically, the physician places a finger either on or between the ribs while using the other hand to monitor the contralateral ribs, again with the patient supine (Fig 3 ). The physician should note symmetry of paired rib motion during the respira-

Figure 3. Evaluating rib motion.

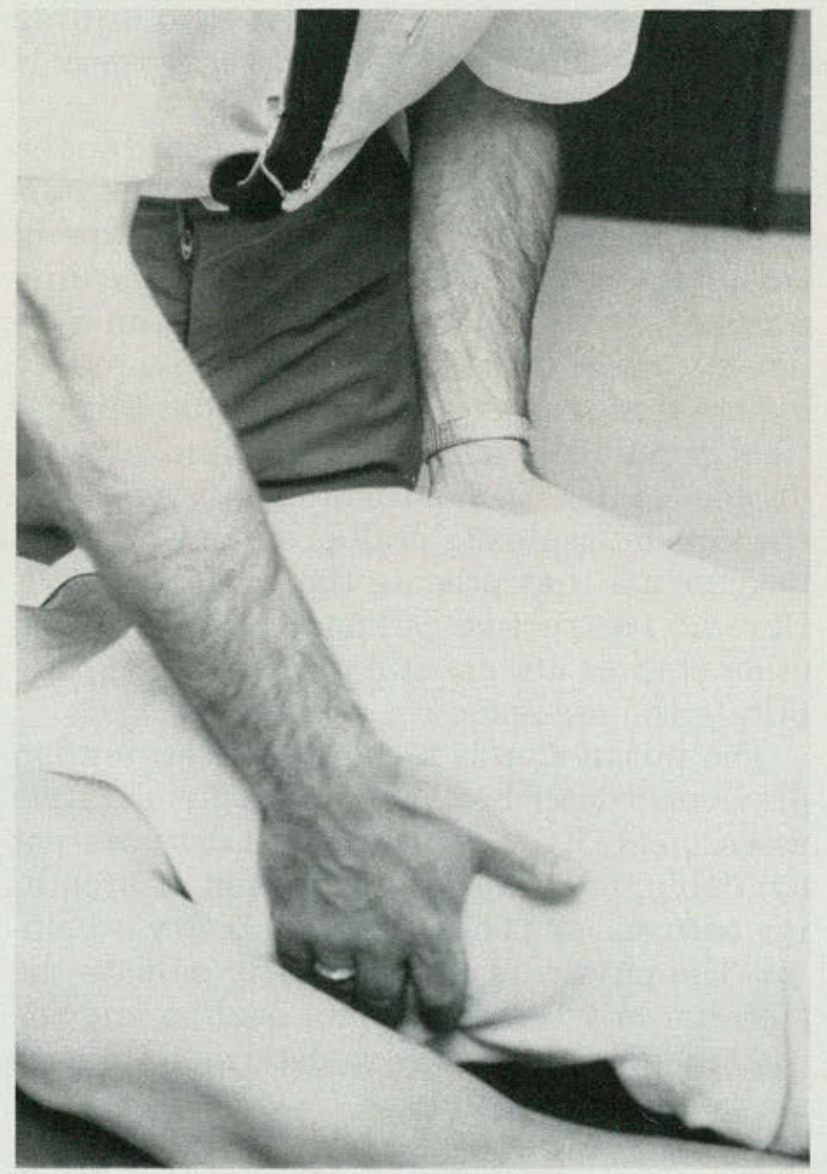


Figure 4. Indirect procedure for rib dysfunction, front view.

tory cycle, or in the case of asymmetry, record the phase of the respiratory cycle in which the asymmetry appeared. With practice, the physician will be able to monitor three or four ribs on both sides simultaneously.

Patients who have had median sternotomy almost always need treatment of multiple areas of somatic dysfunction in the rib cage. Treatment need not be complicated, but it should be atraumatic. The use of indirect ${ }^{7}$ rib technique is particularly valuable because it allows the simultaneous treatment of intercostal muscle spasm and rib articulations.

A technique approach used by Sutherland and outlined by Kimberly ${ }^{8}$ is appropriate for this initial phase of treatment (Figs 4 and 5). The patient sits on one side of the table, and the physician sits on the opposite side. The physician contacts the lesioned rib with the index and middle fingers of one hand along the anterior end of the rib, while positioning the index and middle fingers of the other hand along the posterior angle of the rib. The physician places the thumbs of both hands end to end on the rib at the midaxillary line. The patient straightens the spine and leans sideways toward the physician until making firm contact. The patient is instructed to rotate his or her torso toward the side opposite of contact until the physician feels that the palpated rib head is slightly free from its demifacets.

On instruction, the patient partially inhales and then exhales to allow the physician to determine which respiratory phase permits the greatest freedom from tension. Again, as instructed, the patient assumes the respiratory phase of maximum freedom and holds his or her breath for as long as possible. The physician adjusts his or her contacts to maintain maximum freedom from tension, and holds this position until the rib is free of restriction. Several breaths may be necessary to attain complete freedom of motion. To be effective, this treatment approach may need to be repeated

Figure 5. Indirect procedure for rib dysfunction, rear view.
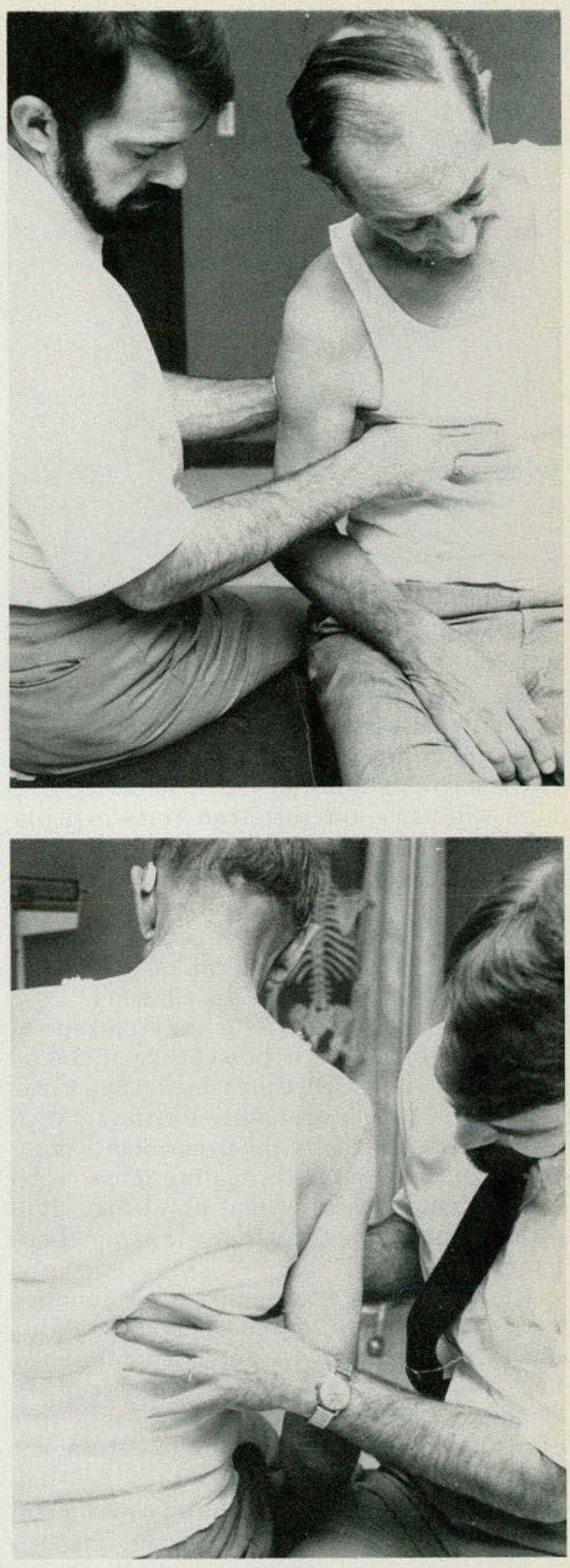
Figure 6. Evaluating and treating fascial restrictions of the thoracic inlet.

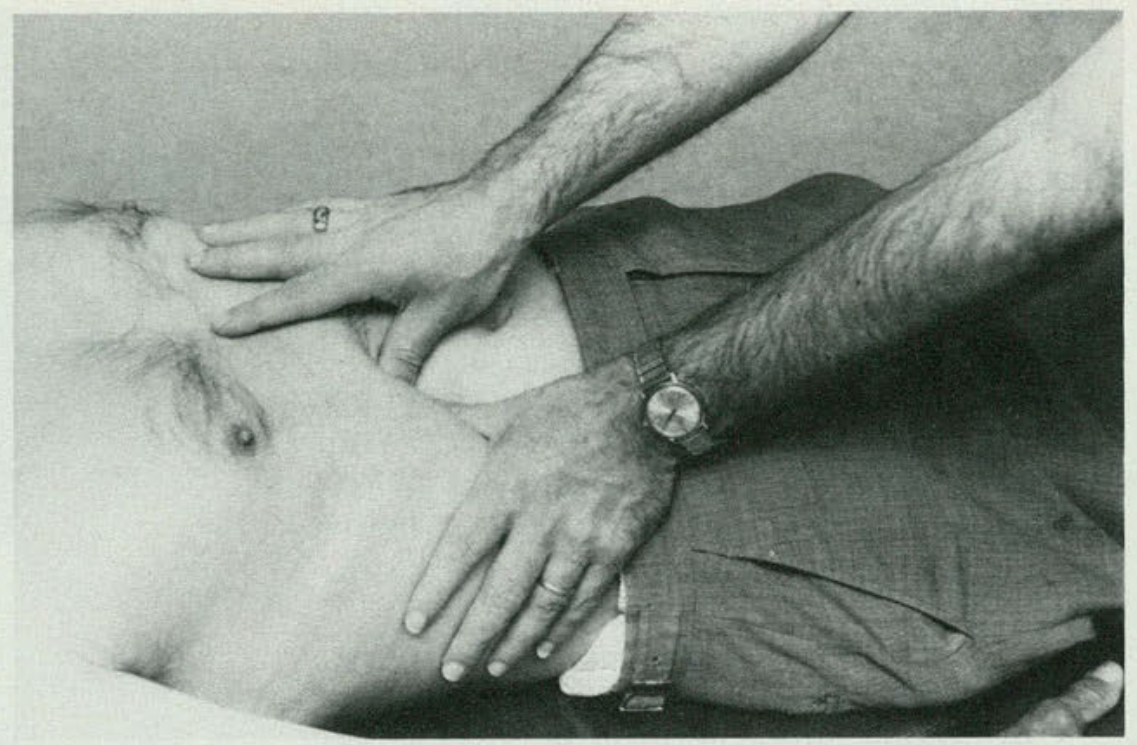

for several weekly visits. It also tends to restore normal sympathetic function through the affected thoracic segments.

The thoracic lateral chain ganglions of the sympathetic division of the autonomic nervous system lie on the anterior surface of the rib heads. Wide anterior retraction of the sternum will cause the costotransverse articulation to act as a fulcrum to lever the rib heads anteriorly from their articulations with the demifacets. Such action may cause injury to the capsule of the rib head and supporting ligaments and create compression of the sympathetic ganglions lying on the rib head.

With healing, scar tissue may entrap the ganglions or create hypomobility of the affected rib. Because rhythmic motion due to respiration is the natural state for the thoracic sympathetic ganglions, the hypomobility of the ribs that develops with healing must represent a distortion in normal physiology, thus altering function of the thoracic sympathetic ganglions.

Disturbed thoracic sympathetic function may be manifested as a vasomotor instability and increased sweat gland activity of the head, neck, thoracic region, or upper extremities. Commonly, patients complain of vague arm pain that does not correspond to dermatome reference patterns. Many times these pain patterns are referable to sympathetic vasomotor disturbances affecting the upper extremities.
Somatic dysfunction of the cervical and thoracic vertebrae is almost always present in the early postoperative period. Thoracic somatic dysfunction is probably due to a combination of direct trauma and somatosomatic reflexes. Somatic dysfunction of the cervical vertebrae is probably related to positioning for intubation and reflexes through the phrenic nerve. Although it is acceptable at this early stage to use direct-action high-velocity techniques for the cervical vertebrae, such techniques should be avoided in the thoracic vertebrae. Initially, it is preferable to use muscle energy ${ }^{9}$ or indirect procedures for thoracic somatic dysfunction.

In the immediate postoperative period, special attention should be given to the diaphragm. Dysfunction will be obvious as distorted breathing patterns and decreased depth of excursion. In addition to traction injury of the diaphragm due to wide retraction of the divided sternum and the lower six ribs, there may be disruptions of the diaphragm's attachments to the xiphoid process of the sternum. The patient may be unable to take a deep inspiration due to pain in the diaphragm. Gentle palpation beneath the inferior border of the rib cage will reveal contraction of narrow slips of fibers of the diaphragm (Fig 6).

At this stage, treatment consists of the physician's gently inserting his or her thumb or fingertips below the costal margin while the 

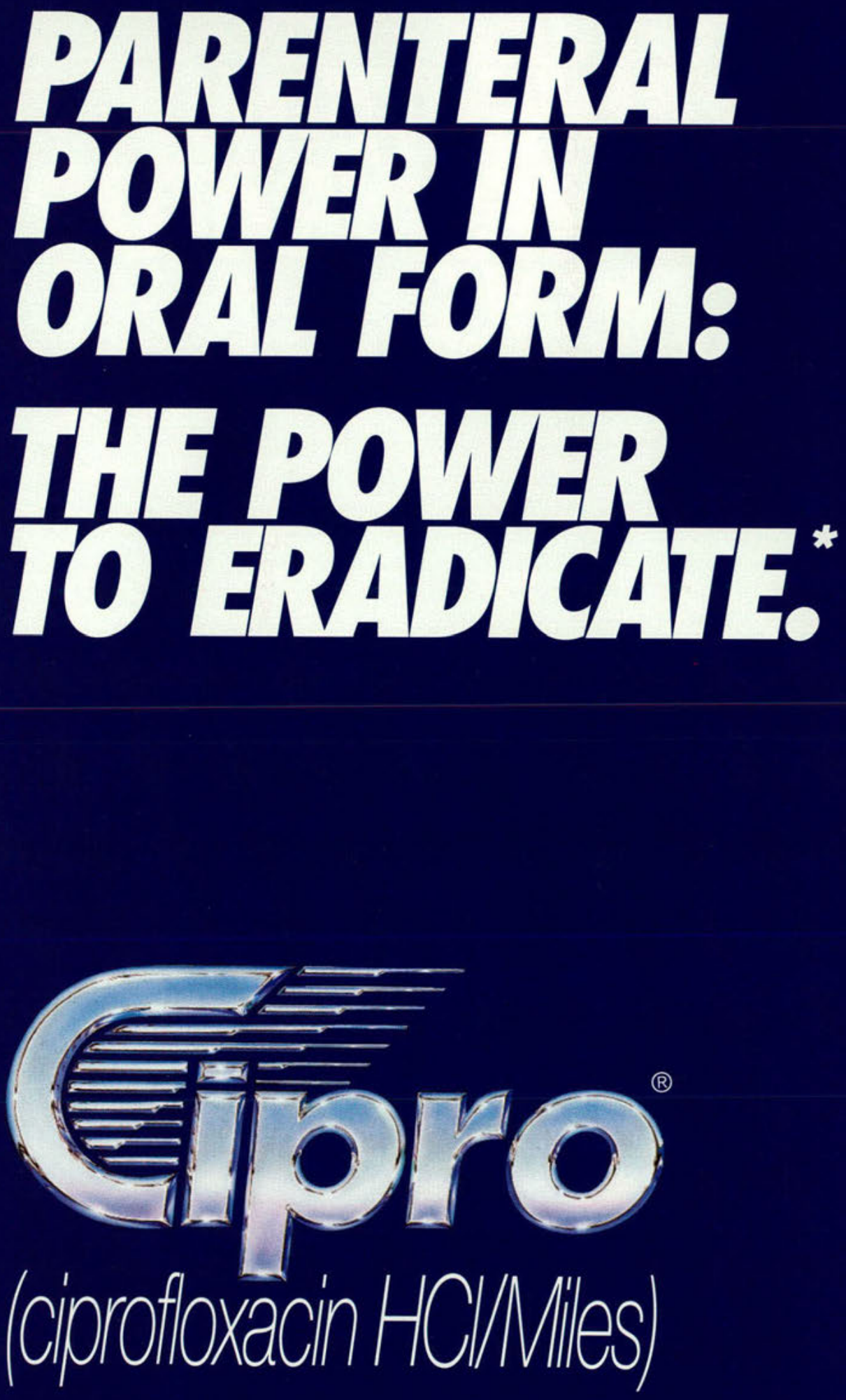

- Achieves $96 \%$ favorable clinical response (resolution + improvement) of infections due to susceptible strains of indicated pathogens. See indicated organisms in prescribing information. 


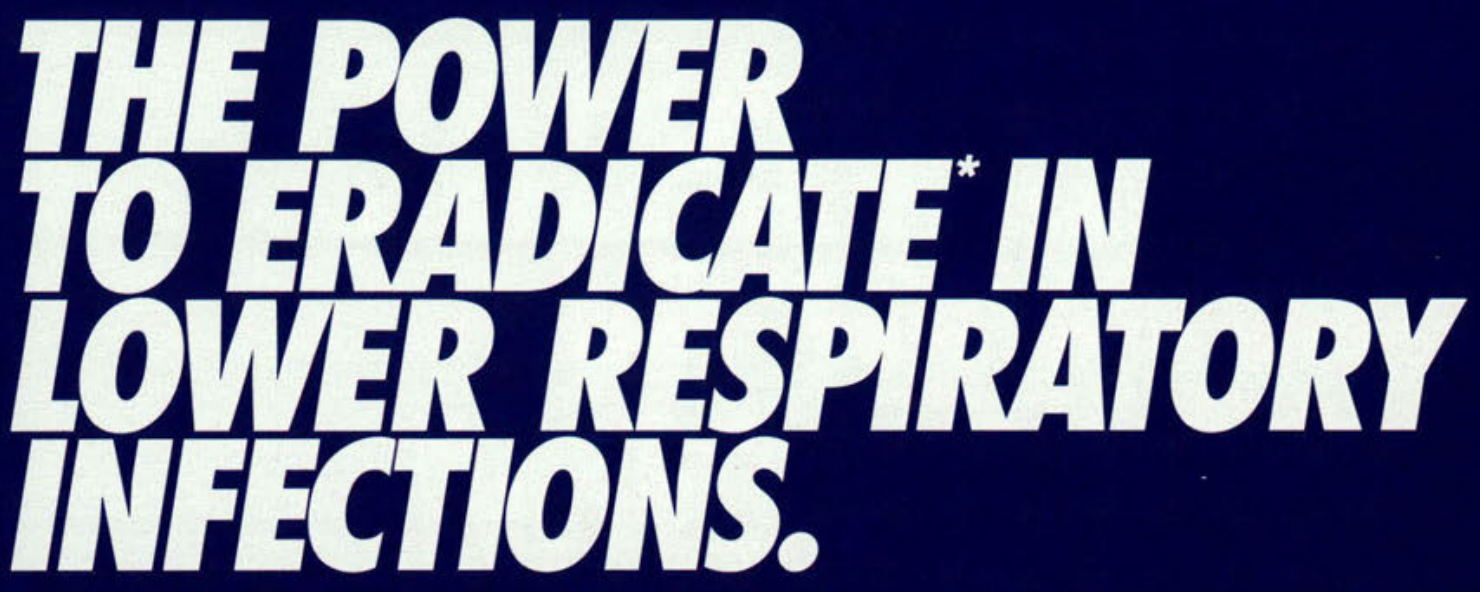

Power that achieves $96 \%$ favorable results in lower respiratory infections. ${ }^{1 \star \dagger}$

Power that destroys pneumopathogens in vitro that are not covered by cefaclor, cephalexin, or ampicillin. ${ }^{2-4 *}$

Power that demonstrates significant activity even against organisms resistant to other agents such as $\mathrm{H}$. influenzae and the Enterobacteriaceae. ${ }^{1,5-7 \star}$

Power that's well tolerated and is comparable in cost with cefaclor and amoxicillin/clavulanate potassium. ${ }^{8}$

NOTES: Concurrent administration of ciprofloxacin with theophylline may lead to elevated plasma concentrations of theophylline and prolongation of its elimination half-life. This may result in increased risk of theophylline-related adverse reactions. If concomitant use cannot be avoided, plasma levels of theophylline should be monitored and dosage adjustments made as appropriate.

Antacids containing magnesium hydroxide or aluminum hydroxide interfere with the absorption of ciprofloxacin, resulting in serum and urine levels lower than desired; concurrent administration of these agents with ciprofloxacin should be avoided.

A history of hypersensitivity to ciprofloxacin is a contraindication to its use.

A history of hypersensitivity to other quinolones may also contraindicate the use of ciprofloxacin.

CIPRO* SHOULD NOT BE USED IN CHILDREN, ADOLESCENTS, OR PREGNANT WOMEN.

\section{The eradicator:*}
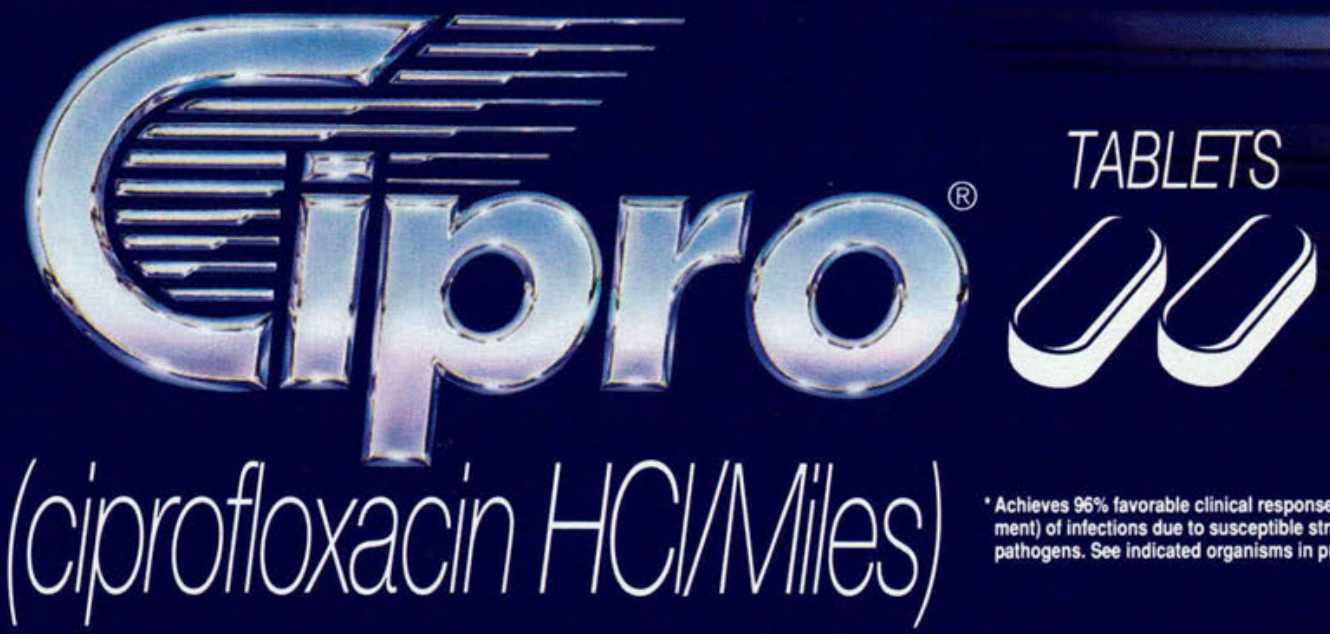

'Achieves $96 \%$ favorable clinical response (resolution + improvement) of infections due to susceptible strains of indicated

pathogens. See indicated organisms in prescribing information. 



\section{声 (ciproforoxacin HCMMlies)}

\section{CIPRO ${ }^{\star}$ TABLETS}

(ciprofloxacin $\mathrm{HCI} /$ Miles)

BRIEF SUMMARY

CONSULT PACKAGE INSERT FOR FULL PRESCRIBING INFORMATION IMDICAMONS AND USAGE

Cipro* is indicated for the treatment of infections caused by susceptible strains of the designated microorganisms in the conditions listed below: Lower Respiratory Intections caused by Escherichia coli, Kebsiella pneumoniae, Enterobacter cloacae, Proteus
mirabilis. Pseudomonas aeruginosa, Haemophilus influenzae, Haemophilus parainfluenzae, and Streptococcus pneumoniae.
Strin and Skin Structure Infections caused by Escherichia coli. Klebsiella pneumoniae. Enterobacter cloacae. Skin and Skin Structure Infections caused by Escherichia coli, Klebsiella pneumoniae, Enterobacter cloacae.
Proteus mirabilis. Proteus vulgaris. Providencia stuartii, Morganella morganii. Citrobacter freundiv, Pseudomonas Proteus miraballs, Proteus vulgaris, Providencia stuartit, Morganella morganiw, Gitrobacter treund
aeruginosa. Staphylococcus aureus. Staphylococcus epidermidis, and Streptococcus pyogenes.

aeruginosa. Staphylococcus aureus. Staphylococcus epidermidis, and Streptococcus pyogenes.
Bone and Joint Infections caused by Enterobacter cloacae. Serratia marcescens. and Pseudomonas aeruginosa. Urinary Tract Infections caused by Escherichia coll, Klebsiella pneumoniae, Enterobacter cloacae. Serratia mar. cescens, Proteus mirabilis. Providencia retigeri. Morganella morganin, Citrobacter diversus. Citrobacter freunditi. cescens, Proteus mirabilis. Providencia rettgeri, Morganella morganu, Citrobacter
Pseudomonas aeruginosa. Staphylococcus epidermidis, and Streptococcus faecalis.

Intectious Diarnhea caused by Escherichia coli (enterotoxigenic strains). Campylobacter jejuni, Shigella flexneri, and Shigella sonner" when antibacterial therapy is indicated

\section{CONTRAINDICAMIONS}

A history of hypersensitivity to ciprofloxacin is a contraindication to its use. A history of hypersensitivity to other quinolones may also contraindicate the use of ciprofloxacin.
WARMINGS

CIPROFLOXACIN SHOULD NOT BE USED IN CHILDREN. ADOLESCENTS, OR PREGNANT WOMEN. The oral administration of ciprofloxacin caused lameness in immature dogs. Histopathological examination of the weight-bearing joints of these dogs revealed permanent lesions of the cartiage. Related drugs such as nalidixic acid, cinoxacin, and norlloxacin also produced erosions of cartilage of weight-bearing joints and other signs of arthropathy in immature
animals of various species (SEE ANIMAL PHARMACOLOGY SECTION IN FULL PRESCRIBING INFORMATION). PRECAUTIONS

General: As with other quinolones, ciprofloxacin may cause central nervous system (CNS) stimulation, which may lead to tremor, restlessness, lightheadedness, confusion, and rarely to hallucinations or convulsive seizures. Therefore, ciprofloxacin should be used with caution in patients with known or suspected CNS disorders, such as severe cerebral arteriosclerosis or epilepsy, or other lactors which predispose to seizures (SEE ADVERSE REACTIONS). Anaphylactic reactions following the first dose have been reported in patients receiving therapy with quinolones. Some reactions were accompanied by cardiovascular collapse. loss of consciousness, tingling. pharyngeal or facial
edema, dyspnea. urticaria, and itching. Only a few patients had a history of hypersensitivity reaction. Anaphylactic edema, dyspnea, urticaria, and itching. Only a few patients had a history of hypersensitivity reaction. Anaphylactic
reactions may require epinephrine and other emergency measures. Ciprofloxacin should be discontinued at the first sign of hypersensitivity or allergy

Severe hypersensitivity reactions characterized by rash, fever, eosinophilia, jaundice, and hepatic necrosis with fatal outcome have been reported rarely (less than one per million prescriptions) in patients receiving ciprofloxacin along with other drugs. The possibility that these reactions were related to ciprofloxacin cannof be excluded. reaction Crystals of ciprofloxacin have been observed rarely in the urine of human subjects but more frequently in the urine
of laboratory animals (SEE ANIMAL PHARMACOLOGY SECTION IN FULL PRESCRIBING INFORMATION). Crystalluria related to ciprofloxacin has been reported only rarely in man, because human urine is usually acidic.
Patients receiving ciprofloxacin should be well hydrated, and alkalinity of the urine should be avoided. The recommended daly dose should not be exceeded

Ateration of the dosage regimen is necessary for patients with impairment of renal function (SEE DOSAGE AND ADMINISTRATION)

As with any potent drug. periodic assessment of organ system functions, including renal, hepatic, and hematopoietic function, is advisable during prolonged therap;

Orug Interactions: As with other quinolones, concurrent administration of ciprofloxacin with theophylline may lead to elevated plasma concentrations of theophylline and prolongation of its elimination half-life. This may result in
increased risk of theophylline-related adverse reactions. If concomitant use cannot be avoided, plasma levels of theophylline should be monitored and dosage adjustments made as appropriate.

Quinolones, including ciprotloxacin, have also been shown to interfere with the metabolism of cafteine. This may lead to reduced clearance of cafteine and a prolongation of its plasma hall-life.

Antacids containing magnesium hydroxide or aluminum hydroxide may interfere with the absorption of ciproflox. acin resulting in serum and urine levels lower than desired; concurrent administration of these agents with ciprofloxacin should be avoided

Concomitant administration of the nonsteroidal anti-inflammatory drug fenbuten with a quinolone has been reported to increase the risk of CNS stimulation and convulsive seizures

Probenecid interteres with the renal tubular secretion of ciprofloxacin and produces an increase in the level of ciprofloxacin in the serum. This should be considered if patients are receiving both drugs concomitantly.

As with other broad-spectrum antibiotics, prolonged use of ciprofloxacin may result in overgrowth of nonsuscepti. ble organisms. Repeated evaluation of the patient's condition and microbial susceptibility testing is essential. If superinfection occurs during therapy, appropriate measures should be taken.

Information for Patients: Patients should be advised that ciprofloxacin may be taken with or without meals. The preferred time of dosing is two hours after a meal. Patients should also be advised to drink fluids liberally and not take antacids containing magnesium or aluminum

Patients should be advised that ciprofloxacin may be associated with hypersensitivity reactions, evén following a single dose, and to discontinue the drug at the first sign of a skin rash or other allergic reaction.

Ciprofloxacin may cause diziness or lightheadedness; therefore patients should know how they react to this drug before they operate an automobile or machinery or engage in activities requiring mental alertness or coordination. Patients should be advised that caprofloxacin may increase the effects of theophylline and caffeine

Carcinogenesis, Mutagenesis, Impairment of Fertility: Eight in vitro mutagenicity tests have been conducted with ciprofloxacin and the test results are listed below

Salmonella/Microsome Test (Negative)

E. coli DNA Repair Assay (Negative)

Mouse Lymphoma Cell Forward Mutation Assay (Positive)

Chinese Hamster V 79 Cell HGPRT Test (Negative)

Syrian Hamster Embryo Cell Transtormation Assay (Negative)

Saccharomyces cerevisiae Point Mutation Assay (Negative)

Saccharomyces cerevisiae Mitotic Crossover and Gene Conversion Assay (Negative)

Rat Hepatocyte DNA Repair Assay (Positive)

Thus, two of the eight tests were positive, but the results of the following three in vivo test systems gave negative (thulit

Rat Hepatocyle DNA Repair Assay

Micronucleus Test (Mice)

Long-term carcinogenicity studies in rats and mice have been completed. After daily oral dosing for up to 2 years. there is no evidence that ciprofloxacin had any carcinogenic or tumorigenic effects in these species.

Pregnancy - Pregnancy Category C: Reproduction studies have been performed in rats and mice at doses up to 6 times the usual daily human dose and have revealed no evidence of impaired fertility or harm to the fetus due to gastrointestinal disturbances resulting in maternal weight loss and an increased incidence of abortion. genicity was observed at either dose. After intravenous administration, at doses up to $20 \mathrm{mg} / \mathrm{kg}$. no maternal toxic was produced, and no embryotoxicity or teratogenicity was observed. There are, however, no adequate and we
controlled studies in preonant women. SINCE CIPROFIOXACIN. UKKE OTHER DRUGS IN ITS CLASS. CAUS controlled studies in pregnant women. SINCE CIPROFLOXACIN. LIKE OTHER DRUGS IN ITS CLASS. CAUS
ARTHROPATHY IN IMMATURE ANIMALS. IT SHOULD NOT BE USED IN PREGNANT WOMEN (SEE WARNINGS) ARTHROPATHY IN IMMATURE ANIMALS, IT SHOULD NOT BE USED IN PREGNANT WOMEN (SEE WARNINGS) Nursing Mothers: It is not known whether ciprofloxacin is excreted in human milk, however, it is known t ciprofloxacin is excreted in the milk of lactating rats and that other drugs of this class are excreted in human $m$
Because of this and because of the potential for serious adverse reactions trom ciprofloxacin in nursing infants Because of this and because of the potential for serious adverse reactions trom ciprofloxacin in nursing infants
decision should be made to discontinue nursing or to discontinue the drug. taking into account the importance of

drug to the mother.

Pedratric Use: Patients under the age of 18 were not included in the clinical trials of ciprofloxacin beca ciprofloxacin as well as other quinolones causes arthropathy in immature animals. Ciprolloxacin should not be us in children or adolescents (SEE WARNINGS)

\section{ADVERSE REACTIONS}

Ciprofloxacin is generally well tolerated. During clinical investigation, 2,799 patients received 2,868 courses of drug. Adverse events that were considered likely to be drug related occurred in $7.3 \%$ of courses, possibly related primarily involving the gastrointestinal system $(1.5 \%)$, skin $(0.6 \%)$, and central nervous system $(0.4 \%)$ Tho events typical of quinolones are italicized

The most frequently reported events, drug related or not, were nausea $(5.2 \%)$, diarrhea (2.3\%), vomiting (2.0 abdominal pain idiscomfort $(1.7 \%)$, headache $(1.2 \%)$, restlessness $(1.1 \%)$, and rash $(1.1 \%)$

Additional events that occurred in less than $1 \%$ of ciprofloxacin courses are listed below

GASTROINTESTINAL: (See above), painful oral mucosa, oral candidiasis, dysphagia, intestinal perforatio gastrointestinal bleeding

CENTRAL NERVOUS SYSTEM: (See above), dizziness, lightheadedness, insomnia, nightmares, halluci tions. manic reaction, irritability, tremor, ataxia, convulsive seizures.

aise. anorexia, phobia, depersonalization, depression, paresthesia. a erythema nodosum

Alergic reactions ranging from urticaria to anaphylactic reactions have been reported (SEE PRECAUTIONS ALES: blurred vision, disturbed vision (change in color perception, overbrightness of light: decreased visual acuity, diplopia, eve pain, tinnitus, hearing loss, bad taste.

MUSCULOSKELETAL: joint or back pain, joint stiffness, achiness, neck or chest pain, flare-up of gou RENALUROGENITAL: inters

bleeding, vaginitis, acidosis. myocardial infarction, cardiopulmonary arrest, cerebral thrombosis.

RESPIRATORY; epistaxis, laryngeal or pulmonary edema, hiccough, hemoptysis, dyspona, bronchospas pulmonary embolism.

Most of the adverse events reported were described as only mild or moderate in severity. abated soon after drug was discontinued, and required no treatment.

In several instances, nausea, vomiting. tremor, restlessness, agitation, or palpitations were judged by vestigators to be related to elevated plasma levels of theophyline possibly as a result of a drug interaction w ciprofloxacin

Other adverse events reported in the postmarketing phase include anaphylactoid reactions. Stevens-Johns syndrome, exfoliative dermatitis, toxic epidermal necrolysis, hepatic necrosis, postural hypotension, possible ex erbation of myasthenia gravis, confusion, dysphasia, nystagmus, pseudomembranous colitis, dyspeps flatulence, and constipation. Also reported were agranulocylosis; elevation of serum triglycerides, sery cholesterol, blood glucose, serum potassium; prolongation of prothrombin time; albuminuria; candiduria, vagin candidiasis; and renal calculi (SEE PRECAUTIONS)

Adverse Laboratory Changes: Changes in laboratory parameters listed as adverse events without regard to dr relationship:

Hepatic-Elevations of $\mathrm{A}$

serum bilirubin $(0.3 \%)$

Choiestatic jaundice has been reported.

platelets $(0.1 \%)$, pancytopenia $(0.1 \%)$

Renal-Elevations of Serum creatinine $(1.1 \%)$. BUN $(0.9 \%)$

CRYSTALLURIA, CYLINORURIA, AND HEMATURIA HAVE BEEN REPORTED

Other changes occurring in less than $0.1 \%$ of courses were: Elevation of serum gammaglutamyl transferas levation of serum amylase, reduction in blood glucose, elevated uric acid, decrease in hemoglobin, anem beeding diathesis, increase in blood monocytes, and leukocylosis. OVERDOSAGE

Information on overdosage in humans is not available. In the event of acute overdosage, the stomach should emptied by inducing vomiting or by gastric lavage. The patient should be carefully observed and given supporti
treatment. Adequate hydration must be maintained. Only a small amount of ciprofloxacin $(<10 \%)$ is removed fro treatment. Adequate hydration must be maintained.

the body after hemodialysis or peritoneal dialysis.
DOSAGE AND ADMINISTRAMION

The usual adult dosage for patients with urinary tract infections is $250 \mathrm{mg}$ every 12 hours. For patients $w$ complicated infections caused by organisms not highly susceptible, $500 \mathrm{mg}$ may be administered every 12 hours Lower respiratory tract infections, skin and skin structure infections, and bone and joint infections may be treate
with $500 \mathrm{mg}$ every 12 hours. For more severe or complicated infections. a dosage of $750 \mathrm{mg}$ may be given every with 50 .

The recommended dosage for infectious diarrhea is $500 \mathrm{mg}$ every 12 hours

In patients with renal impairment, some modfication of dosage is recommended (SEE DOSAGE AND A MINISTRATION SECTION IN FULL PRESCRIBING INFORMATION)

$$
\text { HOW SUPPLIED }
$$

Cipro* (ciprofloxacin HCUMiles) is available as tablets of $250 \mathrm{mg}, 500 \mathrm{mg}$, and $750 \mathrm{mg}$ in bottles of 50 , and in Un Dose packages of 100 (SEE FULL PRESCRIBING INFORMATION FOR COMPLETE DESCRIPTION).

Relerences: 1. Data on file, Miles Inc. Pharmaceutical Division. 2. Physicians' Desk Reference*. 43rd ed. Orade NN: Medical Economics Co inc; 1989:891, 1168, 1441, 2098. 3. Wollschlager CM, et al. Controlled, comparative stud of ciprofloxacin versus ampicilin in treatment of bacterial respiratory tract infections. Am J Med. 1987;82(sup 4A) :164-168. 4. Feist H, et al. Comparative study of ciprofloxacin and cetalexin in the treatment of patients with low. respiratory tract infections. In: Neu HC. Weuta H, eds. Ist International Ciprofloxacin Workshop. Amsterdar Excerpta Medica; 1986:265-267. 5. Parry MF. Ouinolone resistance-trends in U.S. susceptibility data. I Ciprofloxacin: Major Advances In Intravenous and Oral Quinolone Therapy. Scientific Program and Abstracts
Naples, Florida: 1989:21. 6. Sanders CC, et al. Overview of preclinical studies with ciprolloxacin. Am J Mec

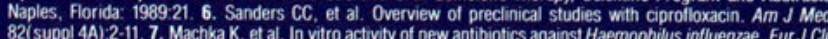
82(suppl 4A):2-11. 7. Machika K, et al. In vitro activity of new antibiotics aga:
Microbial Infect Dis. 1988;7:812-814. 8. Redbook Update. 1989,8(5):4,6.7.

-Achieves $96 \%$ tavorable clinical response (resolution + improvement) of intections due to susceptible strains of indicat For further information, contad the Miles Information Service: 1-800-642-4776. In VA, coll colled: 703-391-7888.

COMMITTED TO THERAPEUTIC EFFICIENC

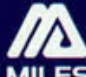

Miles Inc

Pharmaceutical Divisio

MILES

West Haven, CT 065

CO9299 MiL-eos 
patient maintains full exhalation. The physician applies and maintains light pressure to the contracted fibers while the patient inhales fully. A Valsalva maneuver by the patient also will help to stretch the involved fibers. As the patient exhales, the physician's fingertips follow the diaphragm upward and maintain this position as the patient inhales again and performs another Valsalva maneuver. The physician maintains pressure through several respiratory cycles until observing release of tension. It will be necessary to repeat this approach weekly until stubborn areas of contraction are released. For treatment of somatic dysfunction of the lumbar vertebrae, either muscle energy or indirect techniques may be used to release the origin of the diaphragm.

Early in the treatment sequence, specially trained physicians should evaluate the primary respiratory mechanism. The author has found through experience that the sacrum will demonstrate restrictions more often than the cranium, possibly as a result of disruption of fascial continuity, disturbed respiratory patterns, or positioning or transfer maneuvers during surgery (either singly or in combination). The disturbance of respiratory patterns is probably the most likely cause. Spasm and direct injury to the diaphragm alter the tension on its lumbar attachments. Tension is usually noted in the lumbosacral region in the early stages of treatment, resulting in inhibition of motion of the sacral base; however, a common pattern of restriction has not been observed.

Some patients who have no evidence of brachial plexus injury complain of achy pain in the upper extremities, often accompanied by mild swelling in the posterior axillary fold. This combination indicates a lymphatic drainage problem, in which case the physician should assess the tissue preference patterns of the upper arm and the forearm independently. The physician should combine and hold these tissue preference patterns with slight compression through the elbow and shoulder until he or she perceives the tension relaxing. This maneuver may have to be repeated at subsequent visits to provide complete relief.

One should anticipate the presence of myofascial trigger points, formed as a result of the mechanical tissue trauma combined with hypothermia during surgery, in patients who have undergone median sternotomy. Such trigger points are most likely to be located in the pectoral, the trapezius, and latissimus dorsi muscles. By using the Fluori-Methane sprayand-stretch techniques of Travell and Simons, ${ }^{10}$ the physician can eliminate the majority of these trigger points. Failure to address these points can lead to needless pain and disability. Resistant trigger points usually respond to injection of a local anesthetic agent, followed by stretching of the involved muscle fibers.

Because the treatment plan proposed so far cannot be completed at the patient's first visit or at any single visit during the treatment sequence, the physician should treat the areas of greatest need first, and then repeat treatment at subsequent visits. In the time that remains during any given visit, the physician can evaluate and treat areas with secondary need. One should grade treatment to patient age, condition, and stage of healing. For the first 8 to 10 weeks after surgery or until there has been functional union of the sternum and any possible rib fractures, the physician should avoid using high-velocity techniques except for the cervical vertebrae.

\section{Late postoperative treatment plan}

The use of high-velocity techniques has increasing value after the third postoperative month. The wide retraction of the median sternotomy places significant forces on the costotransverse and costovertebral joints, with injury to their capsules and supporting ligaments. This injury appears to be worse in older patients, barrel-chested patients, and patients with fixed rib cages. Hypermobility of costotransverse and costovertebral joints on the initial evaluation of the rib cage motion should lead the examiner to suspect this type of injury. By the third month of healing, scar formation may lead to progressive hypomobility of these joints, at which time the use of thrusting techniques achieves better results.

Problems with lymphatic drainage of the diaphragm, rib cage, and upper extremity are likely to be of concern earlier in the postoperative course. However, by the third or fourth postoperative month, there may be evidence of faulty lymphatic drainage in the head and neck. Persistent puffiness in the lower neck 


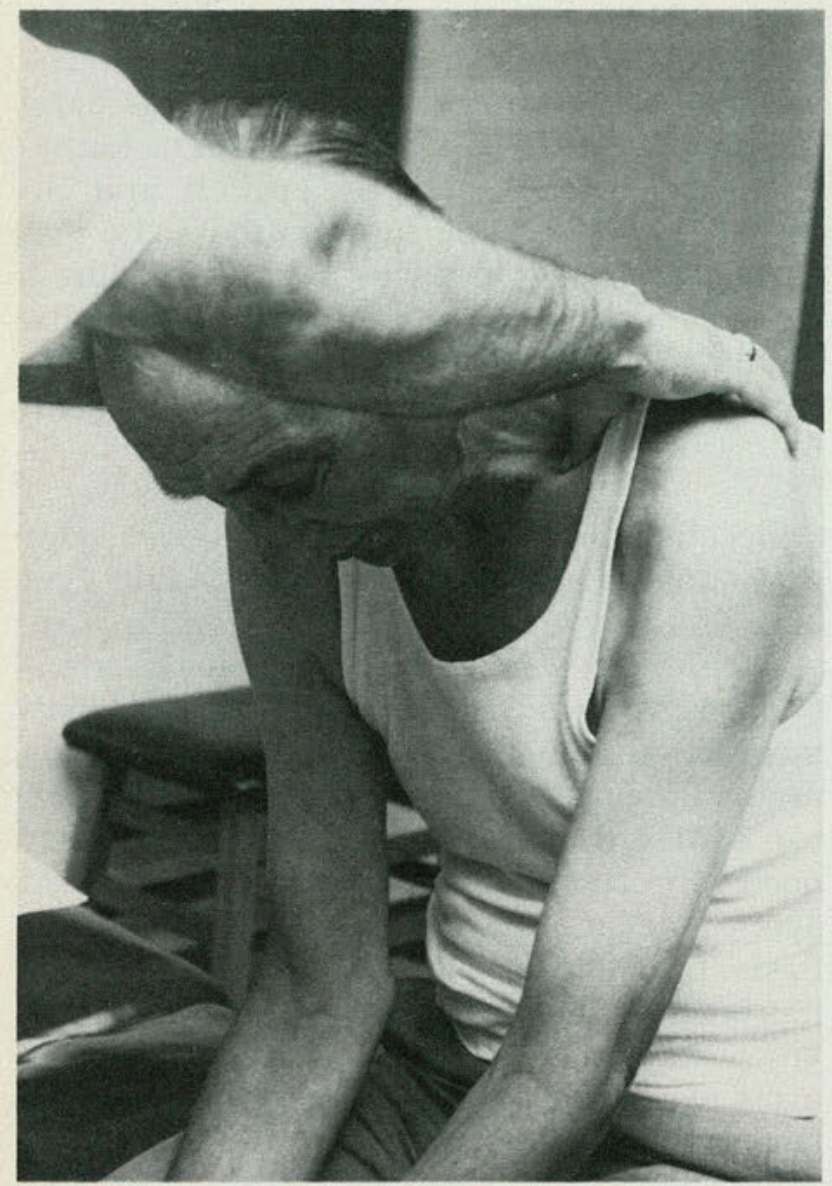

Figure 7. Treating fascial restrictions of the thoracic inlet.

and supraclavicular fossae with achy pain in the neck should alert the physician of such lymphatic drainage problems. The physician should treat somatic dysfunction of the first four thoracic vertebrae and their ribs, clavicles, and sternum. It is possible to stretch the fascias of the lower cervical vertebrae and thoracic inlet by placing the patient in the sitting position, with the head bent forward (Fig 7). The physician places his or her thumbs deeply into the supraclavicular fossae while the patient exhales and bends his or her head and neck farther forward. On instruction, the patient then takes a deep breath, sits up straight, and backward bends the head and neck at the height of inspiration. This technique is intended to lift the anterior cervical fascia.

\section{Adjunctive treatment}

On surgical dismissal, the only advice the patient receives regarding nutrition and life- style is to maintain a low-fat diet, to resume ambulation progressively, and to cease smoking. There is at least a theoretic basis for the use of antioxidants, which should be given consideration. The lowered oxygen tension of surgically traumatized tissues favors the formation of scar tissue over normal tissue. The daily administration of 1000 units of mixed tocopherol vitamin $\mathrm{E}$ for 6 months may be beneficial. The use of a multivitamin B complex is also advised. Because $30 \%$ of the population will have a rise in blood pressure in response to $1 \mathrm{~g}$ of vitamin $\mathrm{E}$, blood pressure should be monitored frequently. If blood pressure does become elevated, the dose of vitamin $\mathrm{E}$ is reduced to 300 to 400 units, supplemented with 50 to $100 \mu \mathrm{g}$ of selenium, which has a synergistic effect. ${ }^{11}$

Because the most difficult obstacle is the maintenance of upper thoracic vertebral and rib mobility during healing, the patient should be instructed in the home use of an exercise bar. Such bars are available at sporting goods stores, or they can be fabricated from parts obtained from any hardware store. The bar should be placed high enough in a doorway to enable the patient to suspend his or her body weight supported by the arms twice daily for 30 seconds. The traction thus applied through the anterior and posterior shoulder muscles and their attachment to the upper ribs will aid mobilization.

One patient has reported that while he was performing this exercise, he felt a tearing sensation behind the sternum and was immediately able to take a deeper breath. He undoubtedly had provoked a breakdown of retrosternal adhesions. His experience gives some indication of the effects of this exercise on all tissues of the upper region of the body. Some of my patients have been voluntarily continuing this exercise for 3 or more years because of its benefits. Patients should begin such exercises at the third postoperative month and continue them for as long as they perceive benefit. One unanticipated benefit of median sternotomy should be mentioned. As previously stated, the acute trauma to the thoracic vertebrae and rib cage renders the thoracic cage hypermobile for 3 to 4 months. Gradually, by the seventh postoperative month, the 
thoracic cage becomes hypomobile as the scar retracts. This "window of opportunity" allows definitive correction of somatic dysfunction of the thoracic vertebrae and rib cage that may have proved refractory to treatment preoperatively. Longstanding and chronic somatic dysfunction of the thoracic vertebrae and rib cage may yield to treatment during this interval.

\section{Report of case}

A 68-year-old male osteopathic physician underwent a quadruple coronary bypass graft procedure via a median sternotomy approach. On the first visit about 3 weeks postoperatively, his chief complaint was sternal, upper thoracic, and cervical pain. He had a 60 -pack per year smoking history and a 20-year history of gouty arthritis.

Neuromusculoskeletal examination revealed obvious increase in thoracic kyphosis as a result of pain on standing erect; chronic rib restrictions of the mid and upper rib cage, extending from T-4 to T-8, secondary to old rib fractures; gouty arthritis in the cervical, thoracic, and lumbar spine; and chronically shallow respiratory excursion. Incomplete healing of the median sternotomy was evident with crepitus to slight compression of the sternal margins. The fascias and tissues overlying the sternum had a pronounced counterclockwise preference pattern. The patient had pain on moderate inhalation, and he had multiple wound drainage sites in the subxiphoid region. Although these drainage exit wounds were dry and healing well, they indicate the invasion of the diaphragm necessary to drain both pleural cavities and the mediastinum.

Treatment was started by the use of indirect fascial techniques directed to the sternum and posterior region of the thorax. Indirect technique was used to mobilize restriction in the cervical, thoracic, and lumbar regions of the spine and the sacrum. The patient was placed on a daily regimen of antioxidant therapy consisting of 400 units of vitamin $\mathrm{E}$, in mixed tocopherol form, supplemented by 50 to $100 \mu \mathrm{g}$ of selenium. This patient had previously had an adverse reaction-an elevation in resting blood pressure - to dosages of vitamin $\mathrm{E}$ in excess of 1000 units per day.

The patient was seen weekly over the next 2 months. At the second visit, all rib heads were articulated to assess mobility and possible soft tissue damage to the articulations themselves. Indirect treatment was again directed to the sternal and cervical fascias, as well as to the upper thoracic and midthoracic regions of the spine, the sacrum, and rib cage.

By the third visit, the patient's sternal pain had resolved except for some residual pain along the xiphoid process. The same fascial pattern was evident at the sternum but was resolving, and the right arm was beginning to show some signs of edema. He had increasing tension in the right crura of the diaphragm. Treatment was aimed at unwinding fascial patterns in the right arm, axilla, and shoulder region as well as being directed at the sternal fascial pattern and to the diaphragm to stretch restricted and scarred regions.

Approximately 2 months postoperatively, the patient began to show signs of trigger points with pain radiating into the arms and into the neck. It is uncertain whether this trigger phenomena had been present since the surgical insult or had been the result of increasing activity in the postopertive period. Pectoral and supraspinatus trigger points were elicited and treated with spray-andstretch technique. Articulatory treatment, consisting of moving joints through their range of motion, was instituted in the thoracic and lumbar regions of the spine.

The patient continued to improve slowly on weekly visits. At each visit, he expressed new symptoms, which were readily relieved by addressing sternal, thoracic, and cervical areas of somatic dysfunction. By the second month, the patient had pain radiating into the anterior aspect of the neck and jaw, which was feared to be an anginal variant. Continuing work on trigger points in the trapezius and pectoral regions resolved this pain pattern. In the second month, high-velocity procedures were directed to the cervical and lumbar regions of the spine. Increasing manipulative activity was directed toward springing and articulatory treatments for the restricted rib cage, and efforts were continued to stretch the forming scar tissue in the diaphragm.

By the end of the second month, more rapid progress was being made and the patient began a biweekly treatment schedule. Efforts were now aimed more toward the thoracic region of the spine and ribs than they had been previously. The right crura of the diaphragm continued to be restricted in its excursion, and the rib cage began showing increasing hypomobility. By the middle of the third postoperative month, muscle energy procedures were started for somatic dysfunction of the rib cage and thoracic region of the spine.

Progress continued, and the patient began on a monthly treatment schedule by the beginning of the fourth postoperative month. At this time, the patient had slight cervical pain on arising, which subsided spontaneously with light activity. The patient remarked that this was typical of his longtime gouty arthritis. Although I continued to fo- 
cus treatment on restricted rib cage mechanics, resolution of the diaphragmatic pattern was beginning to be evident. By this time, the fascial preference pattern of the sternal region was greatly diminished, and there was no evidence of edema in the cervical or supraclavicular regions or upper extremities.

By the end of the fourth postoperative month, I began judicious use of high-velocity/low-amplitude procedures directed to the thoracic region of the spine and the rib cage. The patient had minimal symptoms and was sleeping soundly through the night, whereas he had had a history of awakening at 4 or $5 \mathrm{AM}$ for a number of years because of his gouty arthritis. The patient was now sleeping routinely until $7 \mathrm{AM}$. Somatic dysfunction of the thoracic region of the spine and rib cage, previously refractory to high-velocity force, was easily mobilized at this time. (The preexisting gouty arthritis had always been a deterring factor in the use of high-velocity manipulation in this patient.) The hypermobility of costal articulations was beginning to be reversed at this stage, and every effort was made to maintain proper anatomic relationships and physiologic function during the late stages of scar formation.

By the fifth postoperative month, the patient was asymptomatic. It was evident that scar tissue was retracting because active mobilization of thoracic and rib somatic dysfunction suddenly became more difficult. In addition to the use of direct technique, the use of the home exercise bar was instituted. The patient derived so much benefit from dependent weight hanging from this bar at home that he still uses this exercise periodically as an aid to maintaining proper function and mobility of the thoracic vertebrae of the spine and rib cage and associated fascial structures.

Since the sixth postoperative month, the patient, at his own request, has continued to receive treatment on a near monthly basis. The patient could have been dismissed from regular care any time after the sixth postoperative month, but he feels that the periodic benefits of osteopathic manipulative treatment, in terms of both subjective and objective functioning parameters, justify maintaining a regular treatment schedule. Most patients are dismissed from regular care sometime between the sixth and seventh postoperative month and appear to have no long-term sequelae.

\section{Conclusion}

The osteopathic manipulative treatment protocol described here may benefit a segment of the growing number of patients who are undergoing surgical procedures via the median sternotomy approach. The treatment plan addresses such potential sequelae of this surgical approach as hypomobility and somatic dysfunction of the thoracic region of the spine and rib cage, disruption of fascial patterns through the mediastinum, back pain, and limited or distorted dysfunction of the diaphragm.

1. Johnson MD, Michael R: Median sternotomy for resection of pulmonary metastases. $J$ Thorac Cardiovasc Surg 1983;85:516-522.

2. Rimm AA: Trends in cardiac surgery in the United States. $N$ Engl J Med 1985;213:119.

3. Detailed diagnoses and surgical procedures for patients discharged from short-stay hospitals, 1983. National Health Survey, series 13, No. 82, March 1985, p 165.

4. Harjala A, Jarvinana A: Postoperative median sternotomy dehiscence. Scand J Thorac Cardiovasc Surg 1983;17:277-281.

5. Vander Salm TJ, Cereda JM, Cutter BS: Brachial plexus injury following median sternotomy. J Thorac Cardiovasc Surg 1980;80:447.

6. Zink GJ, Lawson WB: An osteopathic structural examination and functional interpretation of the soma. Osteopathic Annals 1979;7:12-19.

7. Project on Osteopathic Principles Education: Glossary of osteopathic terminology. JAOA 1982;552-567.

8. Kimberly PE (ed): Outline of Osteopathic Manipulative Procedures. Kirksville, Mo, Kirksville College of Osteopathic Medicine, 1980, p 132.

9. Mitchell FL Jr, Moran PS, Pruzzo NA: An Evaluation \& Treatment Manual of Osteopathic Manipulative Procedure, ed 2. Valley Park, Mo, Fall 1973.

10. Travell JF, Simons DG: Myofascial Pain and Dysfunction: The Trigger Point Manual. Baltimore, William \& Wilkins, 1983, p 63.

11. Synergistic relationship of selenium and vitamin E, Medinote. JAOA 1985;85:85:350, 351 . 